\title{
The European (R)evolution of Wireless Digital Networks
}

\author{
Wireless systems engineering is developing into a more conscious search for \\ the best common culture for multiple users in a real environment.
}

Jens C. Arnbak

JENS CHRISTLAN ARNBAK is professor of tele-information techniques at Delft University of Technologv.

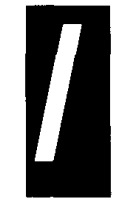

n 1979, the author was invited to the chair of Radio Communications at Eindhoven University of Technology in the Netherlands. Several friends and professional associates questioned the sense of accepting this offer. "Radio is dead. Broadband optical fibre is the future," said the experts.

I wondered if I should trust their kind advice and found five reasons not to. First, during a decade spent in planning international satellite services, I had noted an emerging trend towards more flexible support of end-users by small maritime and landmobile terminals. The most conspicuous example was probably the small dish immediately unfolding in all the foreign locations visited by the roving U.S. Secretary of State, Henry Kissinger. The new individual access links were different from the traditional multiplexed satellite trunk connections; indeed optical cables looked poised to replace in the 1980s. Second, a wireless access network could connect a new subscriber in most urban and rural areas to the public network at lower investment cost than any local loop requiring digging. Third, would the majority of subscribers really ask for the vast individual link capacity offered by installing optical subscriber loops, noting that standard video distribution could be supported by satellite (radio again!), possibly in tandem with existing local cable television (CATV) networks? Fourth, the Nordic Mobile Telephone (NMT) cellular standard was at that time being developed in close cooperation between different PTTs and competing manufacturers in Denmark, Finland, Iceland, Norway, and Sweden. NMT demonstrated a joint drive in European countries towards (inter-)national cellular networks. The divided and divesting United Stateslooked less able to develop and follow a common strategy for mobile networking, even though Bell Laboratories had played a leading role in the initial development of the novel cellular technology in the early 1970s. And finally, the new mobile satellite and cellular networks would offer an opportunity to teach total communication systems engineering. This would seem a dire necessity in most European electrical engineering departments, where the traditional academic separation of 'switching systems', 'transmission systems,' and 'enabling technologies' often leads to more focus on (sub-)system capacity than on system capabilities to meet users' needs.

\section{Back to the Future}

$\boldsymbol{T}^{\text {hese }}$ - personally biased - observations were made almost 15 years ago. Many things look different now, including my own university affiliation and responsibilities. Still, the general emphasis of radio communications designs has shifted away from maximizing the capacity of single links limited by Gaussian noise and available bandwidth, towards optimizing the capabilities (including, but not limited to the capacity) of multi-user networks. The decisive interference now seldom comes from outside, but is produced by authorized users of the very same wireless network. Users thus share an interest in developing and adhering to the best possible protocols and standards for allocating the joint network resources. Accordingly, wireless systems engineering is developing into a more conscious search for the best common culture for multiple users in a real environment. The capacity, spectrum efficiency, and cost-effectiveness of modern radio networks can no longer be won from nature (or an adversary) in a classical pursuit of individual gain.

This development has been commercially reinforced by the recent business trend in the computer and defence sectors away from "selling the high-tech products that you can make" toward "making high-tech products that you can sell." It has become more important to consider the different requirements and "cultures" of new communities using wireless networks, including their needs for innovation of regulatory conditions and standards. In this article, some of the related European developments of two different personal communication services, digital cellular telephony and mobile data networks, are compared with each other and with 
developments in the United States. The related agendas for R \& D of wireless technologies in the past decade are also reviewed and compared.

In 1992, the new standard for Pan-European digital cellular telephony known as GSM [1] saw its first operational successes. The name GSM originated early in the 1980s as the French acronym for Groupe Special Mobile. This international working group was tasked by most European PTT administrations to develop a standard for cellular networks allowing international roaming across the many European borders. A truly Pan-European standard also provides economies of scale in mass production of hand-held and car terminals. In the early $1980 \mathrm{~s}$, these two objectives were seen as the most critical success factors for achieving a much larger penetration of mobile telephone services in Europe. Below, we shall discuss a third factor, the introduction of competition in the monopolistic European markets - a cultural import from the United States.

Although the GSM standard provides digital circuits to mobile terminals, alternative specialized wireless data networks for mobile computer communications were put into operation much earlier in several European countries. Some essential technology differences between the circuit-switched and packet-switched radio systems, and their relative merits in the increasingly competitive European environment, are also reviewed in this article.

\section{Present Status of European Cellular Telephony}

As of April 1993, 32 operators in 22 countries are committed to GSM. In Europe, this is formally expressed by a Memorandum of Understanding (MoU). The Commission of the European Communities (CEC) ensured that common frequency allocations were made in the $900-\mathrm{MHz}$ band in the 12 member states and in many other European countries. The GSM standard is also rapidly being adopted outside Europe, notably in the Asia-Pacific region in Singapore, Malaysia, India, Hong Kong, and Australia, where a Pan-Asian MoU is being considered, and in the Middle East and Africa. Moreover, Taiwan, Thailand, and New Zealand are considering the GSM standard, or have already imposed it for at least one of their competing cellular networks. Accordingly, the old acronym GSM is nowadays taken to mean Global System for Mobile Communication.

The general advantages of international standards, MoUs, and roaming agreementsfor public mobile telephony are increasingly appreciated by different operators and markets. Where the inception and implementation of such conventions lag behind, subscribers are denied some of the following mutual benefits:

- interoperability with the national public switched telephone network(s), including ISDN.

- connectivity to all mobile users in a carrier's service area, stimulating cooperation between adjacent carriers.

- limiting inflexible cable infrastructure to the backbone network.

- low cost of introducing service-area coverage, i.e., proportional to the initial peak network use (erlang/unit area).

- simple upgrading of network capacity, when and where economically justified, by reducing cell size ('cell splitting').
- by virtue of the third, fourth and fifth benefits above, less economies of scale than in hard-wired networks and, hence, less basis for 'natural monopoly' arguments and for regulation against competition between local network operators [2].

- ability to locate vehicles and roaming user terminals, allowing automatic billing of users away from their home location or own operator.

- international portability of a subscriber identity module (SIM) smart card, authorizing customized personal log-in from compatible foreign terminals and competing networks by inserting the SIM card [1].

Such benefits have caused the growth of mobile communications markets to exceed 60 percent p.a. in many countries. No other telecommunications sector can boast similar growth rates at present. The infrastructure supply market is dominated by the few international manufacturers who combine expertise in both radio transmission and national switching systems. One of these manufacturers, L. M. Ericsson of Sweden, has some 40 percent of the cellularworld market, including a major proportion of the many networks using the American analog standard AMPS. The terminal market, on the other hand, is subject to the typical supply principles and economies of consumer electronics: short product development times and a "killing" competition, due to the eroding profit margins on micro-electronics commodity products. However, the resulting drop in terminal prices now assists in developing the service market much faster than in the past.

At the end of 1992, commercial GSM telephone service was offered in eight countries. The coverage area rolled out in Europe at that time is shown in Fig. 1. The major transit routes in Europe had been reasonably covered within one year after the first GSM service went on air, despite economic recession in most countries and delays in type approval of the first telephone handsets and their complicated software. So far, the service is restricted to voice-type digital circuits, but a simple service allowing transfer of short (i.e., paging-type) alphanumeric messages is also planned. A more complete set of data rates to be supported as bearer services in GSM at a later stage (beyond 1995?) has also been defined [3].

Strikingly, the fastest take-up of GSM has been in Germany, where the national economy has stagnated in the wake of the unification of East and West. After only six months of operation in December 1992, Mannesmann Mobilfunk announced the 100,000 th subscriber to its GSM-network, known as D2. In May 1993, 220,000 users subscribed to D2. Mannesmann's competitor in Germany, Deutsche Bundespost Telekom, holds the national monopoly on both public analog mobile telephony and the fixed telephone network. Some $90 \%$ of all telephone calls to or from mobile users in Germany originate or terminate in the latter, and Telekom's analog cellular network, C1, is already virtually saturated by its 800,000 subscribers. Nevertheless, Telekom'sown GSM-network, D1, is believed to have attracted fewer subscribers than the competing D2 network. The successful marketing of the D2 network services and the associated offer of the 'Handy' portable GSM-terminal at a price of less than $\$ 1400$ (including 15 percent tax, deductible for business users) is clearly designed by D2's American shareholder, Pacific
Strikingly,

the fastest

take-up of

GSM has

been in

Germany,

where the

national

economy has

stagnated

in the wake

of the

unification

of East

and West. 


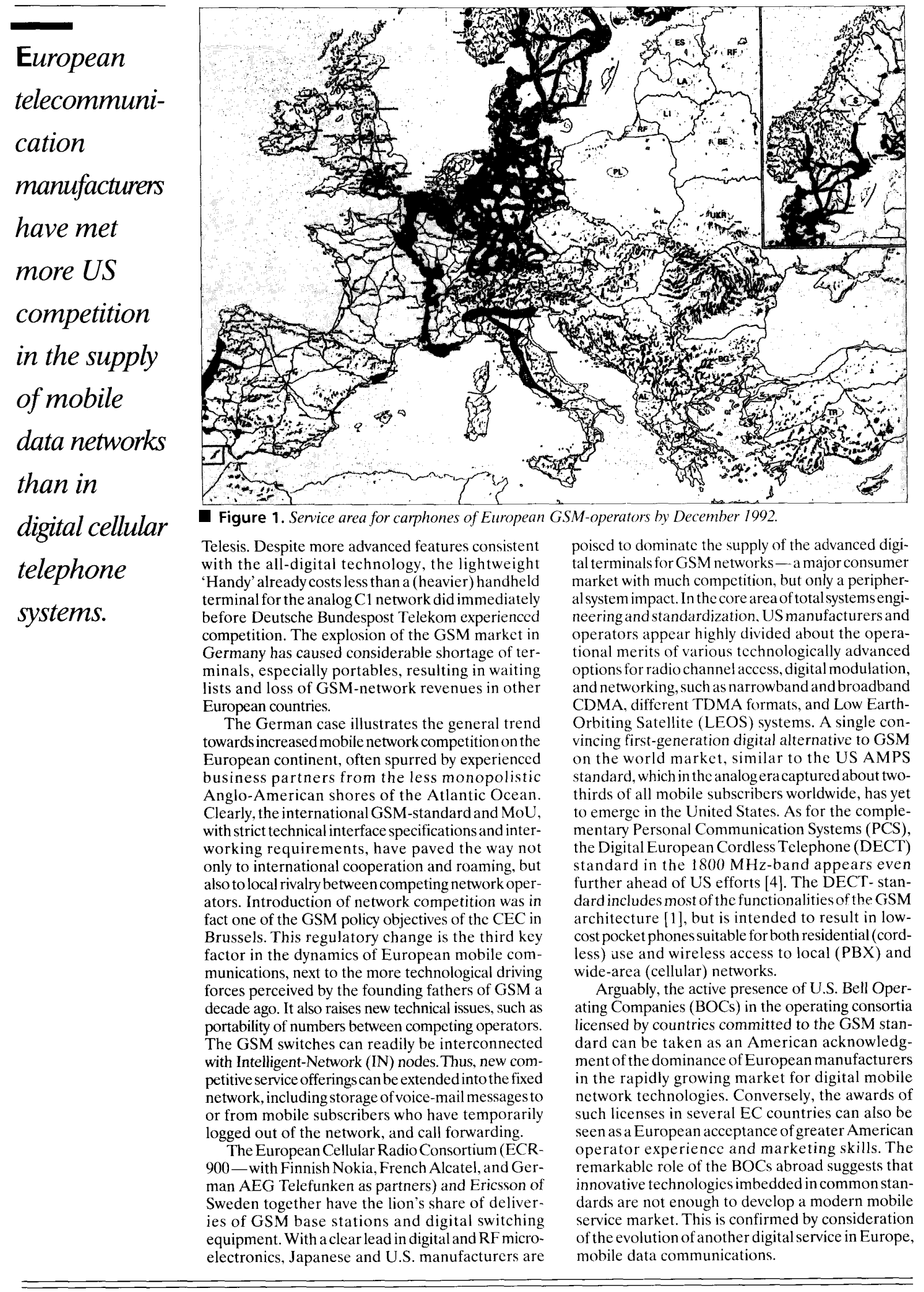




\section{Mobile Data Networks in Europe}

n many European countries, the only possible way to conduct data transmission over public mobile networks is still to attach low-speed voice-band modems to analog radio telephone circuits. Generally, this results in a poor bit error rate due to the signal fading and shadowing, the extra-cellular interference and the handoffs between base stations inherent in mobile systems. Improvement by suitable error-correction codes or protocols is possible, but reduces the throughput of the narrowband radio channel further.

With the advent of the digital GSM, there is a popular belief that mobile computer communications will become easier, cheaper and better. The circuit quality and data rate do indeed improve, but a circuit-switched voice channel is not really suited to any bursty data source, even if using digital transmission. Dialing up (and paying for!) a real-time twoway circuit between end users is quite inefficient for the most frequent modes of mobile computer communications: electronic mail, interactive access to information services, EDI-type computer messaging, dispatch and other types of fleet management, and data "broadcasting." For such applications, the features of classical packet switching appear more desirable: non-blocking access for terminals and the ability to convert data rates and codes within the store-and-forward network. Wide-area flexibility and adaptability to serve computers and terminals with different functions and priorities point toward packet radio networks which can bill by traffic volume, rather than by connect time.

Such mobile data networks operate in several European countries. It is significant to note here that Deutsche Bundespost Telekom in the Spring of 1993 announced that its present experimental pilot mobile data network, Modacom, will be available for full operation in 80 percent of Germany by 1995. This wireless extension of Telekom's own public X.25 packet service to mobile computers such as Laptops, Notebooks, and Palmtops suggests that (at least) one of the major GSM operators in Europe has doubts about the competitiveness of the planned data transmission modes in the GSM network [3] It is expected that the German government will license a second mobile data network later in 1993.

Well ahead of the German Modacom network the Scandinavian PTT-administrations in Sweden, Norway, and Finland introduced the Mobitex Radio Data Standard in the last decade. At that time, Mobitex was supporting only a low-speed packet service at $1200 \mathrm{~b} / \mathrm{s}$ plus an emergency voice service, reflecting the initial use by police patrols, fire brigades, and public utility services to exchange brief command-and-control messages with their headquarters. A decade later, the widespread acceptance of portable computers and the advent of more demanding commercial operators, including RAM Mobile Data in the United States, have pushed the Mobitex data rate up -- and the hybrid voice channel out: the $19.2 \mathrm{kbit} / \mathrm{s}$ data rate defined in [7] is twice that foreseen in GSM [3]. Mobitex based networks are also being introduced in the United Kingdom and the Netherlands by RAM and in France by France Telecom, assisted by Bell South. Again, we note the involvement of U.S. expertise in service provision, even where the network technology and standards are purely European.

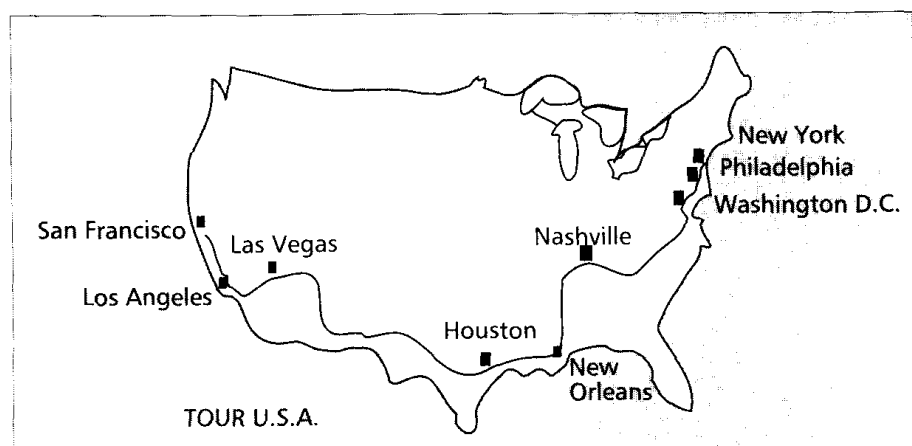

Figure 2. EGA screen dump of route map encoded by differential chain coding into 1200 -byte message $[6]$.

Nevertheless, European telecommunication manufacturers have met more U.S. competition in the supply of mobile data networks than in digital cellular telephone systems. Thus, Motorola's DataTAC technology, based on the RD LAP protocolfor the logical radio link connection, was adopted by Deutsche Bundespost Telekom for Modacom in Germany, by Hutchison in the United Kingdom and Hong Kong, and by the ARDIS companies in the United States and Canada.

To illustrate the possibilities with narrowband mobile data throughputs, imagine the transfer of the simple American route map shown in Fig. 2 to (or from) a mobile terminal. It is possible to encode this image and the associated text as a message string of some 1200 bytes from a graphic tablet or a modern pen computer, using Differential Chain Coding [6]. This message occupies a nominal time of only half a second in a $19.2 \mathrm{~kb} / \mathrm{s}$ packet data channel, considerably less than when dialling up a circuit-switched mobile telephone channel, especially if this were used to interconnect two facsimile terminals using slow runlength codes [6]. Even for the short 1200 byte message string, the time and the signalling overhead to build up the real-time circuit would likely result in a rather unattractive tariff. While a packet-radio protocol could also cause some extra delay, especially during heavy traffic loads, the billing would still correspond to an effective data transfer of only 1200 bytes.

Obviously, the need to link computers in a wireless mode does not only exist outdoor for public networks, but is often driven from the local-area networks at customer premises. The European DECT standard mentioned in the previous section will make data link throughputs up to $1 \mathrm{Mb} / \mathrm{s}$ available, but the limited access time $(50 \mathrm{~ms})$ makes it more suited for wireless linkage of multiple users with considerably lower individual throughputs to a PBX. In the office domain, the United States has more experience with wireless broadband networks than any other country. This gives microcellular wideband systems as Motorola's 18 GHz ALTAIR, meeting the IEEE 802.3 Ethernet standard, and NCR/AT\& T's WaveLAN, using CDMA at $2.4 \mathrm{GHz}$, a competitive edge against the Europeans, when it comes to capacity. However, NCR has performed much of its related research in the Netherlands, where "Bell Labs Europe" is being created. This could result in closer coordination of the wireless-LAN standardization work in the European Telecommunications Standardisatin Institute (ETSI) and IEEE 802.11.

Finally, mention should be made of the present 


\section{European}

harmoniza-

tion is

deemed

essential

to achieve

cross-border

operation

with the

TETRA

standard. work by ETSI on two Trans European Trunked Radio (TETRA) standards, one for pure packet data services to single or multiple destinations, and another also supporting additional circuit-switched data and speech channels (similarly to the initial Mobitex svstems in Scandinavia mentioned above). As we shall see below, a hybrid system cannot be optimized in terms of capacity for both types of services; hybrid systems may nevertheless be required for certain applications, e.g., ones in the transport sector. The European frequency allocations for TETRA are likely to be in the UHF band (parts of $380-400 \mathrm{MHz}, 410-430 \mathrm{MHz}, 450-470 \mathrm{MHz}$ and/or $870-890 / 915-933 \mathrm{MHz}$ ), and to adopt $25 \mathrm{kHz}$ channels for co-existence with existing mobile services. TETRA is thus a typical narrowband data standard. European harmonization is deemed essential to achieve cross-border operation with this second-generation standard, in the interest of international courier services, railroads, and road and river transport companies.

\section{Differences Between Packet Data and Digital Voice Systems}

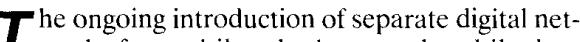
works for mobile telephony and mobile data applications in Europe described above will not come as a surprise to experienced network engineers. In the past, optimum use of classical hard-wired transmission resources motivated separate communications networks and signaling protocols for telephone and for computer traffic, adapted to the different statistical characteristics of the corresponding information sources. Real-time, blocking-type channels for telephone conversations pose other network requirements than does the delayed, but non-blocking exchange of bursty data in computer sessions, e.g., using the X.25 protocol. Where the physical transmission media contribute additional random fluctuations (as in mobile radio due to signal fading and mutual interference), or when the channel capacity is too precious to allow the overhead of ISDN-type service integration (as in mobile radio due to spectrum shortage), further distinctions arise between voice and data networks.

It is the instantaneous blocking probability, as determined by any unacceptable co-channel interference between cells, that determines the optimum design and real-time capacity of optimum cellular telephone networks. Although digital modulation schemes can be made more robust to interference than analog modulation, they still require considerable spacing between cells using the same radio frequencies, in order to avoid unacceptable degradation of voice circuits from time to time. A notable exception is spread-spectrum modulation as used in CDMA cellular systems, which trade carrier bandwidth for processing gain to tolerate a higher co-channel interference. The consequence is a stricter requirement for power control in mobile spread-spectrum telephone systems, in order to reap the theoretical network capacity and spectrum efficiency in the face of the fluctuating interference in the mobile channel [7].

Cellular structures and/or power control algorithms optimized for voice circuits are not optimum for a mobile network of virtual circuits between users who are prepared to accept delays of some data packets. Users of packet protocols are quite will-

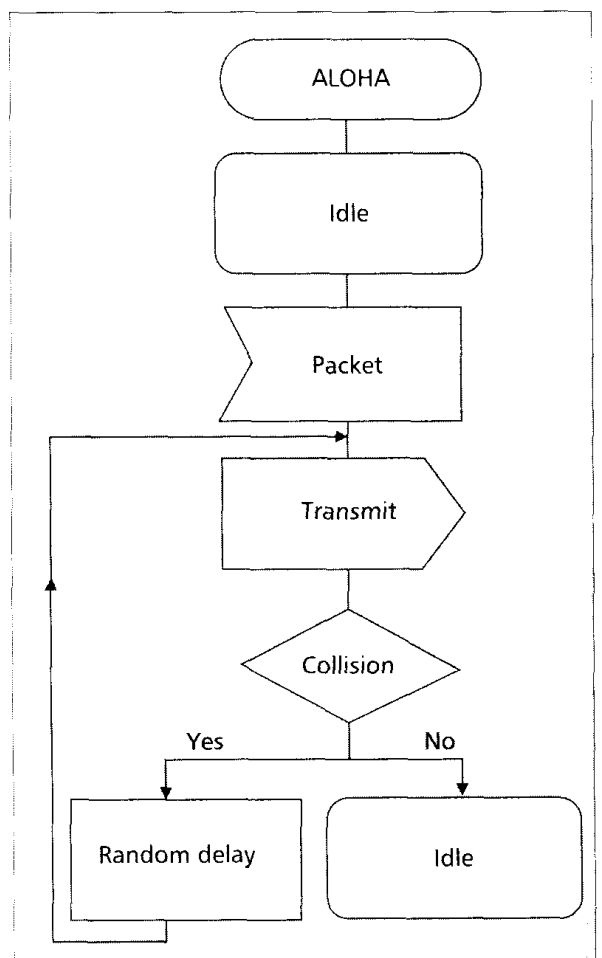

Figure 3. Flow' chan for network access by mobile data terminal using non-slotted ALOHA [11].

ing to accept a significant risk of harmful collisions between coinciding packets, in return for not having to schedule their bursty accesses very strictly or to defer transmission for a long time. Packet protocols are designed to repair any harmful interference experienced between packets simply by retransmission. This contrasts with classical fixed and mobile telephone circuit requirements, which contain substantial a priori guarantees against occasional circuit outages. including those due to mutual harmful interference of radio signals.

When computer users are prepared to gamble against the risk of mutual conflicts in order to reap the benefit of lower average delay [7], they can also adopt a more tolerant attitude to the additional random vagaries of the mobile radio channel. Moreover, users prepared to accept occasional mutual conflicts between their accesses may find it counterproductive to invoke the strict power control assumed in mobile telephone systems. For if access powers are very unequal, there is a higher chance that at least onc of the competitors wins the contest for the receiver than in the event of perfectly balanced signals, which will all annihilate each other in a collision.

\section{Simple Multiple-Access Methods for Mobile Data Networks}

$M$

obile transmitters sending bursty traffic in he form of data packets to a common basestation receiver can, in general, best use some kind of random access. The classical ALOHA protocol, according to which each mobile terminal is frce to offer bursty packets to the channel in accordance with the simple flow diagram in Fig. 3, belongs in this category. It is well known that the 
(ideal) channel throughput can be doubled if active terminals are prepared to synchronize their packet transmissions in to common time slots, such that the risk of partial packet overlap is avoided [7]. With high traffic loads, however, both unslotted and slotted ALOHA protocols become inefficient, since the free competition between all transmitters exposes most of the offered data traffic to collisions and, hence, multiple retransmissions and increasing delays.

To reduce this risk, a transmitter can follow a more cautious strategy. By first listening either to the common radio channel or to the "acknowledgement" return channel from the base station, a transmitter with a data packet can attempt to determine whether the shared radio facilities are already busy. The terminal approach based on the former listening method is known as carrier-sense multiple access (CSMA) [7]. In a realistic mobile channel, the various CSMA protocols may fail to detect ongoing radio transmissions of packets subject to deep fading on the listening path. Therefore, CSMA proves less efficient than in classical hard-wired and satellite networks, where contending terminals are not "hidden" from each other by individually different radio propagation effects. In such circumstances, mobile data terminals can better listen to the common base station, which broadcasts a "busy" signal to acknowledge an incoming transmission and inhibit prospective competitors and/or an "idle" signal to invite transmissions.

In principle, the simplest random-access protocols are inherently unstable, given the standard assumptions of infinitely many users and Poissondistributed offered traffic. In practice, however, realistic ALOHA models based on finite populations of competiting mobile terminals and proper propagation characteristics of the shared mobile channel have good stability properties [8]. Above all, this applies when the common base station receiver can be captured by a stronger packet in the presence of weaker competitors, and provides an incentive not to use too sophisticated random-access protocols in practical low-cost mobile networks.

\section{Receiver Capture Effects in Mobile Data Networks}

n a mobile telephone network, every effort should be made to avoid propagation-induced outages of circuits, once these have been set up for real-time connections. It is a normal goal in both cellular engineering and adaptive power-control schemes to keep the capacity-limiting carrier-to-interference $(\mathrm{C} / \mathrm{I})$ ratios equally high at all receivers throughout a mobile telephone network, in order to secure the prescribed grade of service and voice circuit quality.

Equal C/I-ratios would not necessarily be a proper goal in multi-user packet communications. In 1976 , Metzner showed that utilization of an ALOHA channel can be improved by deliberately introducing differences between the access powers of multiple users competing for a joint receiver [7]. In 1982, the author and one of his graduate students [9] demonstrated this effect in a particular case of a mobile packet radio system, where different propagation losses due to spatially distributed terminals and Rayleigh-distributed signal fluctuations due to multipath fading effects invariably introduce differences

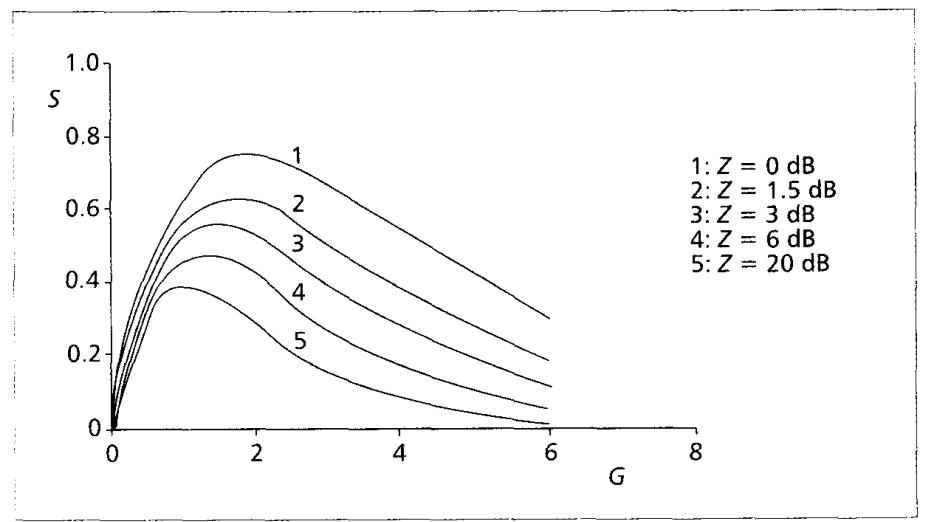

Figure 4. Nomalized throughput of single-cell mobile slotted ALOHA network, assuming uncorrelated Rayleigh fading of all packets and a base-station receiver with capture ratio $\mathrm{Z}[21]$.

in C/I-ratios on the different links. To illustrate the effect of such differences, Fig. 4 shows the normalized throughput, $S$, of a slotted mobile ALOHA channel with offered normalized traffic, $G$, and uncorrelated multipath fading of the different packets. The formula for the multi-user throughput in thisinterference-limited network follows [10]:

$$
S=G \exp \{-G /(1+1 / Z)\}
$$

The capture ratio of the base station, $Z$, indicates its ability to discriminate against a received signal in the presence of a co-channel signal which is $Z$ times stronger. A thorough discussion of the validity of this simple receiver-capture model is found in the Ph.D. thesis of Linnartz [11]. For narrowband digital modulation, typical values for $Z$ may be between $4 \mathrm{~dB}$ and $10 \mathrm{~dB}$; an increase of $S$ results, relative to the case without capture, i.e., when $Z$ is infinite. In the latter case, the standard result

$$
S=G \exp (-G)
$$

for slotted ALOHA in "ideal" (i.e., non-fading AWGN) channels [7] is recovered.

Note that the maximum throughput of (1)

$$
S^{\prime}=(1+1 / Z) \exp (-1)
$$

occurs at increasingly higher offered packet traffic

$$
G^{\prime}=(1+1 / Z)
$$

as the receiver capture ratio $Z$ is decreased. Thus, both greater capacity and betterstability result. With perfect receiver capture $(Z=1)$, equations 2 to 4 would suggest a doubled channel capacity due to Rayleigh fading and receiver capture capabilities. If $Z$ would be allowed to tend to zero as in a spreadspectrum receiver, $S$ would even remain close to the offered traffic $G$ up to a level determined by the "processing gain" $1 / Z$. Within the limitations [11] of this simple model, it does suggest that a combination of ALOHA and modulation with processing gain might support a very efficient randomaccess mobile data network. The absence of fast power control reaps the full contribution of random multipath fading to the random-access game played by the terminals! 


\section{One of}

\section{the most}

interesting

promises of

modern

wireless

communi-

cations

is a lower

initial cost

of connecting

each

subscriber

to the public

network,

largely

independent

of distance.
Impact of the 'Poor' Mobile Radio Channel

$T$ he foundations of packet radio were laid by U.S. researchers, mostly sponsored by military agencies. More emphasis was put on hostile interference and strategies for network survivability, than on optimum self-interfering systems and the random fluctuations of mobile radio channels. For this reason, historical terms like "packet radio" or "packet broadcasting" seldom refer to the typical propagation features of realistic wireless media, but reflect the purely architectural or information-theoretical notion of maximum connectivity among all terminals in a multi-user network. The experimental use of satellite links with their nearly perfect AWGN channels did not stimulate much consideration of real channel impairments, except hard-limiting satellite amplifiers and jamming by an adversary, where appropriate. When terrestrial networks were considered, these were often appropriate to a tactical battlefield scenario, with geographically distributed store-and-forward repeater nodes linked by random paths with fixed, but unknown losses. The desired packet communication modes were generally of the multi-hop type, designed to maximize the progress of packets in particular directions.

As a consequence of this strong research tradition, many researchers still intuitively expect the significant propagation impairments of typical terrestrial UHF/VHF mobile channels to reduce the moderate theoretical throughput of contention protocols ( $S^{\prime}=0.38$ at $G^{\prime}=1$ for slotted ALOHA). However, as discussed above in the previous section, colliding packets with very different ground-wave losses or instantaneous fading levels do not necessarily all annihilate each other, given receiver capture capabilities. Indeed, throughput expressions such as equation 1 for "poor" mobile channels indicate a higher capacity than suggested by the classical studies of contention protocols in 'ideal' noiseless or AWGN channels.

The typical electromagnetic shadowing effects of large obstacles cause slow radio signal fluctuations in the mobile channel; the resulting slow-fading statistics is characterized by a log-normal distribution, with a r.m.s. spread $\sigma$ between $6 \mathrm{~dB}$ and $12 \mathrm{~dB}$. If individual data packets can be assumed to undergo uncorrelated shadowing, this gives rise to more significant capacity increases than does the Rayleigh fading due to multipath propagation. Figure 5 illustrates this for a receiver with moderate capture capability $(Z=6 \mathrm{~dB})$. However, it should be noted that mutual correlation between the signal strengths of individual packets becomes much more likely in the presence of shadowing. Recent progress in the study of this very complicated phenomenon is reported in [12].

A related concern in radio propagation is that of the time constants of the fading fluctuations on links to or from mobile terminals. These time constants have a decisive influence on the throughput of packets. Generally, modelling is much easier if the channel state can be considered "frozen" during any one packet transmission, but completely decorrelated between any two packets [11]. The conditions for this obviously depend on carrier frequency, terminal velocities, packet lengths and the intensity of competing traffic. In general, short

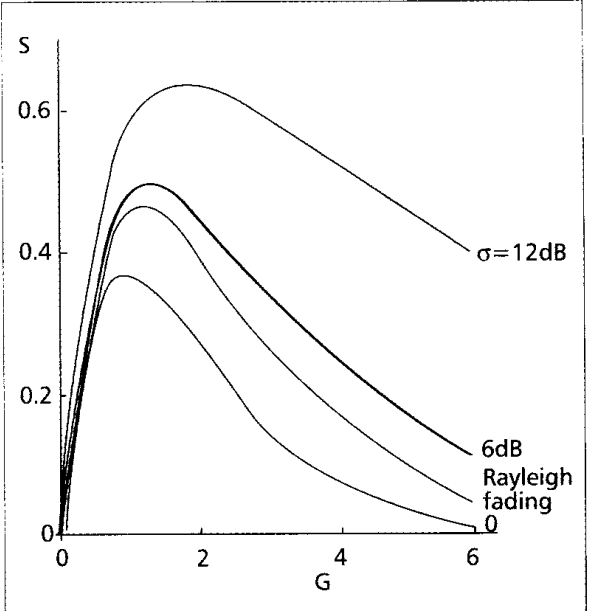

Figure 5. Normalized throughput of single-cell mobile slotted ALOHA network, assuming lognormal shadow fading of all packets with spread $\sigma$, and a base station with capture ratio $Z=6 d B$.

packets profit most from the receiver capture effect described in the previous section, but on the other hand also suffer more from overhead losses in preambles, synchronization, or buffering. Optimal protocol design cannot ignore this trade-off between propagation-induced and higher OSI-level requirements posed by the particular data application.

Note that some of the statistical advantages reaped by packet networking to or from mobile terminals are due to the ergodic properties of the propagation: time and ensemble averages can be interchanged in many calculations, due to the movements of most terminals and the resulting fading fluctuations. Note also that this interchange does not apply to the nearfar effect caused by the classical ground-wave path loss at UHF and VHF. This particular propagation effect creates a deterministic spatial discrimination around each base station [10] which, in effect, decides the effective frequency-reuse distance in a cellular system. The spatial bias can be studied very elegantly using a Laplace transform technique originating from the presence of Rayleigh-fading interferers [11], and can be repaired by a strategy restoring spatial fairness inside the cell by allocating higher retransmission rates for distant contenders.

\section{Cellular Engineering Aspects}

$T$ here is an inherent tolerance against interferers built into all contention-oriented multipleaccess schemes, since any packet failures will ultimately be repaired by protocol measures (retransmissions). This can be expected to lead to considerably smaller optimal cell re-use distances than in narrowband cellular telephone systems. Whether analog or digital, the latter require a considerable spacing between any two cells using the same frequency to ensure sufficient circuit quality. On the other hand, using contiguous "cells" with all base stations accepting the entire available bandwidth may prove better in many cases of packet networking, even in the absence of spread-spectrum processing gains in the base station receivers $(Z>1)$. A considerable chance of capturing a base station from outside its proper cell exists, especially if competition is not too heavy [11]. While this "site diversity" 
can be exploited in mobile data communications to reduce retransmissions of packets and so enhance throughput, it would be indicative of an unacceptable probability of harmful interference between cells in a mobile telephone system! It can thus be postulated that frequency re-use distances should be made much smaller in packet-switched mobile networks than in cellular circuit-switched systems.

Hence, hybrid or integrated cellular voice/data systems may be sub-optimal from a viewpoint of spectrum efficiency and capacity. If voice must be integrated in a computer-oriented cellular network for more than occasional use, it would seem necessary to develop more robust and delay-tolerant ways of packetizing it. Asynchronous transfer mode (ATM) experience maybecome relevant in this context. Conversely, if data packets are supported in a circuitswitched cellular network, inefficiencies in the use of transmission and signalling resources would appear to result. Where cost-based tariff regulations or plain competition apply, specialized mobile data networks therefore look more attractive to all users who attach only little value to the availability of voice transmission in the same network.

\section{Economic and Regulatory Issues}

ne of the most interesting promises of modern wireless communications is a lower initial cost of connecting each subscriber to the public network, largely independent of distance. The access technologies employed in digital cellular radio such as GSM, Personal Communication Systems (PCS) such as first-generation Telepoint systems, the next-generation Cordless Telephone (DECT) and more full-fledged wireless office systems (WOS) using broadband radio LANs, all offer interconnection with public networks at an investment which is mainly determined by the capacity required. This contrasts with the high initial investment of a hard-wired local loop (whether optical or not), the cost of which increases dramatically in rural areas, roughly in inverse proportion to the population density and required traffic capacity! Fig. 6 illustrates these differences schematically [2].

It is the perverse cost structure of the traditional hard-wired access to public networks which caused 'market failure' to occur in many low-traffic rural regions until now and so justified a local operating monopoly for public telephony in most European countries and the United States. However, the novel wireless technologies may help avoiding any need to cross-subsidize the local access from monopoly profits made elsewhere, because costs directly proportional to area capacity remove the classical market-failure problem. In Fig. 6, wireless access to the public infrastructure would appear preferable to (installing new) twisted-pair loops left of the point 1 , and to (installing) optical fibre to the home left of the point 2 . In such circumstances, the economies of scale of the access network might become so marginal that local competition could be allowed under regulatory conditions to ensure fair spectrum allocation, consistent number planning and interconnectivity among the competitors. This is the case for the pan-European GSM-system, thanks to the policy directives from the CEC. Arguably, the recent acquisition of McCaw Cellular Communications by AT\&T can be seen as the re-entry of the divested long-distance carrier into local tele-

\section{$\%$ Telephone subscribers}

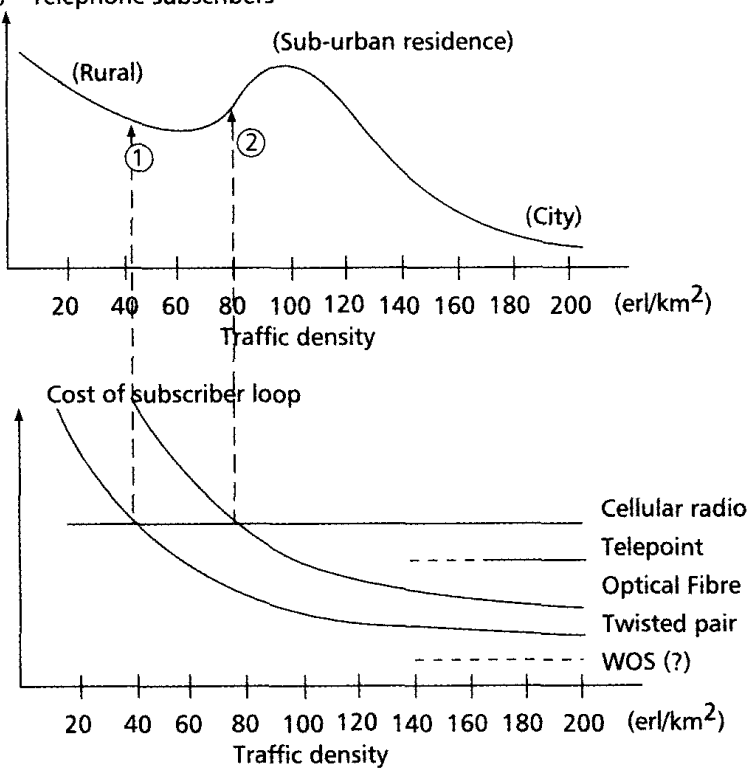

Figure 6. The economy of various access techniques to public telephone network [2]. Above: Subscriber distribution in typical service area, as a function of traffic density. Below: Corresponding costs of different subscriberaccess techniques. Crossover points indicate the transition where cellular access techniques are cheaper than: 1) twisted-pair subscriber loop;2) optical fiber to the home.

phone operations - and hence, as a US example of introduction of competition in a classical monopoly field, using wireless technology.

It is not yet evident on which basis equitabie access by competitors to scarce frequency and number resources can best be granted. In the United States, the FCC has replaced cumbersome administrative hearings by simple lotteries of frequency assignments. This resulted in rapid taking of "windfall profits" by fortunate winners, who simply sold their successful lots immediately after award. This proves that frequencies have a substantial "market" value. Where the Public Purse wishes to enjoy the profit of this, the government must design an auction system and the associated property rights very carefully to avoid being outsmarted by collusions of bidders.

Despite such practical difficulties with auctions of frequencies, it would at least seem desirable to assign some fee for spectrum occupancy. Fairer and more efficient use of scarce resources implies avoidance of extended "free parking" by inactive holders of frequency assignments. Even in the absence of competition, an incumbent holder of frequencies should be given sufficient incentive to vacate or share them for alternative use when not exploiting them fully. Broadcasters outside active operation hours occupy spectrum with considerable value for public and private mobile communications. In the successful European teletext standard, inactive video lines of a TV signal can be used to broadcast or download a substantial amount of data to arbitrary locations inside the coverage area of each TV transmitter network. This is commercially used in several European countries and by Luxembourg's broadcasting satellites ASTRA. Narrowband public datacasting of 
The

development

of novel

wireless

technologies

in the past

15 years has

spurred a

fascinating

drive

towards new

multi-user

systems and

applications

on either side

of the

Atlantic

ocean. traffic information to automobile radios is offered in several European countries, using residual transmit capacity on standard FM-radio carriers in the broadcast band $88 \mathrm{MHz}$ to $108 \mathrm{MHz}$ and the European Radio Data System (RDS) standard [13].

\section{Conclusion: Different Cultural Paradigms.}

7 he development of novel wireless technologies in the past 15 years has spurred a fascinating drive towards new multi-user systems and applications on either side of the Atlantic ocean. But while the enabling technologies are largely the same, some clear differences in the approach and involvement of European and American regulatory standardization bodies can be noted. The stronger European tradition of involvement of public authorities in telecommunications leads to firmer and more widely accepted standards for new public wireless systems, such as GSM, DECT, teletext and RDS. These common standards are proving of great commercial value not only to European manufacturers and system integrators of mobile infrastructure, but also to terminal equipment manufacturers. service operators and users world-wide.

In the United States, the government's role in steering communications system and technology $\mathrm{R} \& \mathrm{D}$ has traditionally been limited to the defense and aerospace sectors. The increasing competition between telecommunication operators now seems to result in an overwhelming 'smorgasbord' of alternative wireless technologies [4]. Frequently, these are based on military spin-offs such as CDMA and packet radio, with as yet no clear market winner in terms of successful publicstandards. On the other hand, the more competitive U.S. attitude to the use of computer and information technology, and the considerable financial resources of the BOCs, prove very powerful in the marketing of the new wireless services in the crumbling European telecommunication monopolies.

Most foundations of packet radio and CDMA were certainly laid in the United States, as evidenced by [7]. However, the shift from military to public multi-user networks required paradigm shifts in research focus which may perhaps have come easier in Europe. Thus, an earlier engineering attention appears in European system studies to the - often counter-intuitive - influence of physical channe impairments on random-access methods, cellular engineering and power control, as reviewed and further developed in [11] and [14]. Implications of other major paradigm shifts of information theory in the event of multiple self-interfering users [15], such as logarithmically unbounded traffic throughput with increased channel load, similar to the "cock- tail-party' shouting effect, and non-applicability of the classical source/channel separation theorem in radio networks, have yet to be fully realized.

In more than one sense, all these changes and differences in economic, regulatory and technological research paradigms illustrate the fact that communication systems engineering is not merely a discipline related to the sciences of nature, but also to the protocols of culture.

\section{References}

[1] Moe Rahnema, "Overview of the GSM System and Protocol Architecture", IEEE Commun. Mag., vol. 31, no. 4, pp. 92-100, April 1993

[2] J. C. Ambak, "Economic and Policy issues in the Regulation of Conditions for Subscriber Access and Market Entry to Telecommunications," in W. F. Korthals Altes et al., eds., Information Law Towards the 21 st Century. (Kluwer, Boston, 1992)

[3] European Telecommunication Standards Institute (ETSI), "Bearer Services Supported by GSM PLMN," Rec. GSM 02.02, Jan. 1990

4] K. Lynch, "U.S. Seen Losing Cellular Advantage," Commun. Weekly. March 22, 1993

[5] K. Parsa, "The Mobitex Packet-Switched Radio Data System," Proc 3rd IEEE PIMRC Symposium, Boston, MA, pp 534-538, 1992

[6] J. C. Arnbak, J. H. Bons, and J. W. Vieveen, "Graphical Correspondence in Electronic-Mail Networks Using Personal Computers, IEEE Journ. Select. Areas. Comm., vol. J-SAC7, no. 2, pp. 257-267, Feb. 1989.

[7] N. Abramson, ed., Multiple Access Communications - Founda tions for Emerging Technologies, (Selected Reprint Volume, IEEE Press, New York, 1993

[8] C. van der Plas and J.P.M.G. Linnartz, "Stability of Mobile Slotted ALOHA Network with Rayleigh Fading, Shadowing and Near-far Effects," IEEE Trans. Vehicular Technol., vol. VT-39, pp. 359-366, Nov. 1990

(9) F. Kuperus and J. Arnbak, "Packet radio in a Rayleigh channel, Electronics Lett., vol. 18, pp. 506-507, June 10, 1982.

[10] J.C. Arnbak and W. van Blitterswijk, "Capacity of slotted ALOHA in Rayleigh fading channel," IEEE Journ. Select. Areas Commun. vol. JSAC-5, pp. 261-269, Feb. 1987

[11] J.P.M. G. Linnartz, "Effects of Fading and Interference in Narrow-band Land-mobile Networks," Ph.D. thesis, Delft University, 1991. (Also published as: J.-P. Linnartz, Narrowband Land-Mobile Radio Networks, Artech House, Boston and London, 1993.)

[12] A. Safak and R. Prasad, "Effects of correlated shadowing signals an channel reuse in mobile radio systems," IEEE Trans. Vehicular Technol., vol. 40, no. 4, pp. 708-713, Nov. 1991

[13] D.S. Chadwick et al., "Communications Architecture for Early Implementation of Intelligent Vehicle Highway System." IEEE Vehicular Technol. Society News, vol 40, no 2, pp 63-70.

[14] J. Zander, "Distributed Cochannel Interference Control in Cellular Radio Systems." IEEE Trans. Vehir 311, 1992

[15] T. M. Cover and J. A. Thomas, Elements of Information Theory, (Wiley, New York, 1991). See in particular Chapter 14: Network Informa tion Theory.

\section{Biography}

Jens Christian ARnbak received Master's and Doctor's degrees from the Technical University of Denmark. From 1972 to 1980 he planned and designed international integrated digital communications networks and satelite systems for NATO at STC in The Netherlands. Between 1980 and 1986 he held a chair of radiocommunications at Eindhoven University of Technology, The Netherlands. Since May 1986, he has been a professor of tele-information techniques at Delft University of Technology, The Netherlands. He has published numerous scientific papers on electromagnetic wave propagation, satellite communication, and packet radio systems and was program chairman of the ICCCIFIP-TC6 conference "ISDNin Europe in 1989 . Since 1982, he has participated invarious telecommunications policy studies commissioned by the Dutch Government, inter alla recommending the new structure and status of the Nethands PT. Hewas a member of the Government Commiteevew it for Dost Penal Code wirh respect to computercine, and of the CounCommitte for Iformaton and Communich Minister's Coordinating Committee for Information and Communication Policy. 